\title{
Effects of sunitinib on the expression of keratin 9 in human keratinocyte cell lines and a 3D skin model
}

\author{
Ayaka Yoshida ${ }^{1,2}$, Kazuhiro Yamamoto ${ }^{1}$, Akane Murakawa ${ }^{1}$, Takahiro Ishida $^{3}$, Tsutomu Nakagawa ${ }^{1}$, \\ Tomoo Itoh ${ }^{2}$, Ikuko Yano ${ }^{1}$
}

${ }^{I}$ Department of Pharmacy, Kobe University Hospita, Japan, ${ }^{2}$ Department of Diagnostic Pathology, Kobe University Graduate School of Medicine, Japan, ${ }^{3}$ R\&D Department, Momotani Juntenkan Ltd., Japan

Background: Hand-foot skin reaction (HFSR) is a common side effect of multiple tyrosine kinase inhibitors. HFSR can lead to dose reduction or the interruption of treatment due to decreasing QOL. The molecular cornification mechanisms of HFSR remain poorly understood. This study aimed to examine the effects of sunitinib on the expression of keratin 9 (KRT9), localized only in the terminally differentiated epidermis of the palmar and plantar skin.

Methods: The expression of KRT9 in a 3D human epidermis tissue model comprising normal human epidermal keratinocytes were evaluated by immunohistochemical staining. The expression of KRT9 and Wnt/beta-catenin-related protein and mRNA in human epidermal keratinocytes ( $\mathrm{HaCaT}$ ) and human skin fibroblast (SF-TY) cell lines were evaluated using western blot and real-time qPCR analyses.

Results and Discussion: HaCaT cells cultured in a lower $\mathrm{Ca}+(0.2 \mathrm{mM})$ medium, but not in a higher $\mathrm{Ca}+(2.0 \mathrm{mM})$ medium, exhibited an enhanced expression of the KRT9 protein and mRNA in a sunitinib concentration-dependent manner. Immunohistochemical imaging revealed that KRT9 expression in the 3D skin model was not enhanced by exposure to sunitinib. 3D skin model consists of a stratified epithelium harboring differentiated keratinocytes that are induced by high $\mathrm{Ca}+$ concentration medium. These results suggested that the $\mathrm{Ca}+$ concentration regulated the enhanced expression of KRT9 by sunitinib in keratinocytes. Moreover, HaCaT cells co-cultured with SF-TY cells exhibited markedly increased expression levels of KRT9 following sunitinib treatment, compared with HaCaT cells alone. SF-TY cells showed that sunitinib enhanced the mRNA levels of Wnt5a and DKK-1, which positively regulate KRT9 expression. Treating skin fibroblasts with sunitinib may increase the expression of KRT9 in keratinocytes via factors related to Wnt/beta-catenin signaling.

Conclusions: The enhancement of KRT9 expression, regulated by $\mathrm{Ca}+$ concentration and fibroblasts, was a mechanism of sunitinib-induced HFSR. 